\title{
BDS/GPS Integrated Positioning Method Research Based on Nonlinear Kalman Filtering
}

\author{
Yan Ma ${ }^{\mathrm{a} *}$, Weilin Yuan ${ }^{\mathrm{b}}$, Huabo Sun ${ }^{\mathrm{c}}$ \\ ${ }^{a}$ China Academy of Civil Aviation Science and Technology, 100028 Beijing, China - may@ mail.castc.org.cn \\ ${ }^{\mathrm{b}}$ China Aero Geophysical Survey Remote Sensing Center for Land and Resources (AGRS), , 100083 Beijing, China - \\ yuan_weilin@126.com \\ ${ }^{\mathrm{c}}$ China Academy of Civil Aviation Science and Technology, 100028 Beijing, China - sunhb@ mail.castc.org.cn
}

\section{Commission IV, WG IV/3}

KEY WORDS: Extended Kalman Filter(EKF), Unscented Kalman Filter(UKF), Particle Filter(PF), Integrated Positioning

\begin{abstract}
:
In order to realize fast and accurate BDS/GPS integrated positioning, it is necessary to overcome the adverse effects of signal attenuation, multipath effect and echo interference to ensure the result of continuous and accurate navigation and positioning. In this paper, pseudo-range positioning is used as the mathematical model. In the stage of data preprocessing, using precise and smooth carrier phase measurement value to promote the rough pseudo-range measurement value without ambiguity. At last, the Extended Kalman Filter(EKF), the Unscented Kalman Filter(UKF) and the Particle Filter(PF) algorithm are applied in the integrated positioning method for higher positioning accuracy. The experimental results show that the positioning accuracy of PF is the highest, and UKF is better than EKF.
\end{abstract}

\section{INTRODUCTION}

With the growing of navigational information resources, the use of each system in combination in order to improve the positioning accuracy and reliability will be the inevitable development of satellite positioning technology. GPS is the most widely used navigation and positioning system currently, which is able to provide high-precision navigation and positioning services for users in worldwide. BeiDou Navigation Satellite System (BDS) is being implemented in China as an independent research and development system. BeiDou global satellite navigation system with 35 satellites will be built around 2020, which will together with GPS, GLONASS and Galileo provide high accuracy as well as reliable navigation and positioning service for the global users. Therefore, BDS/GPS combination navigation and positioning will also be an important development direction of future high-precision positioning. The paper carried out BDS/GPS integrated positioning method based on summary of the current integrated positioning technology research status and analysis the problems and trends. Based on Extended Kalman Filter (EKF), Unscented Kalman Filter (UKF) and Particle Filter (PF), BDS/GPS integrated positioning solution is proposed and constructed as well as the corresponding observation equation and measurement equation.

\section{INTEGRATED POSITIONING PRINCIPLE}

Using the pseudo-range positioning as the mathematical model, the parameters in the formula are shown as following. $\tau^{j} \_$the real time of signal transmitted of Satellite named $s^{j}$; $\tau_{i}$ _the real time when the receiver named ${ }^{T_{i}}$ receives the satellite signal;

$t^{j}$ - the satellite clock time when Satellite $s^{j}$ transmitting the signal;

$t_{i} \ldots$ the receiver clock time when the receiver ${ }^{T_{i}}$ receives the satellite signal;

$\Delta t_{i}^{j} \longrightarrow$ the transmission time of the satellite signal reaches the receiver;

$\delta t^{j}$ _ the clock error of the satellite clock;

$\delta t_{i}$ he clock error of the receiver clock,

Thus,

$$
\left\{\begin{array}{l}
t^{j}=\tau^{j}+\delta t^{j} \\
t_{i}=\tau_{i}+\delta t_{i}
\end{array}\right.
$$

The time of signal transmitted from satellite to the receiver is as following,

$$
\Delta t_{i}^{j}=t_{i}-t^{j}=\tau_{i}-\tau^{j}+\delta t_{i}-\delta t^{j}
$$

In the pseudo-range positioning model, the pseudo-range observation is as below,

$$
\rho_{i}^{j}=c \cdot\left(\tau_{i}-\delta t_{i}\right)-c \cdot\left(\tau^{j}-\delta t^{j}\right)=c \cdot\left(\tau_{i}-\tau^{j}\right)-c \cdot \delta t^{j}+c \cdot \delta t_{i}
$$

As the signal transmits with the speed of light except the ionosphere and troposphere, the geometric distance between

* Corresponding author 
the satellite and observation station is calculated to consider the ionospheric delay $d_{i \text { ion }}^{j}$ and the tropospheric delay $d_{i \text { trop }}^{j}$. Thus, the basic model of the pseudo-range positioning is as following.

$$
\rho_{i}^{j}=R_{i}^{j}\left(t_{i}, t^{j}\right)-c \cdot \delta t^{j}+c \cdot \delta t_{i}+d_{i \text { ion }}^{j}+d_{i \text { trop }}^{j}
$$

$R_{i}^{j}\left(t_{i}, t^{j}\right)$ is the geometric distance between the satellite position of time $t^{j}$ and the receiver position of time ${ }^{t_{i}}$.

$$
R_{i}^{j}\left(t_{i}, t^{j}\right)=\sqrt{\left(x^{j}-x_{i}\right)^{2}+\left(y^{j}-y_{i}\right)^{2}+\left(z^{j}-z_{i}\right)^{2}}
$$

\section{APPLICATION OF NONLINEAR KALMAN FILTER IN BDS/GPS INTEGRATED POSITIONING METHOD}

\subsection{State Equation of Integrated Positioning Method}

The state vector $X$ of the system is transferred form $t_{k-I}$ to $t_{k}$ according to the following dynamic model.

$$
X_{k}=\phi_{k, k-1} X_{k-1}+G_{k-1} W_{k-1}
$$

Among them, $X_{k}=\left[x, y, z, v_{x}, v_{y}, v_{z}, a_{x}, a_{y}, a_{z}\right]$ is the state vector of the system, including three-dimensional position, three-dimensional velocity, as well as threedimensional accelerated velocity.

As the mobile devices are low dynamic in this research, the Singer model is chosen as the motion model. The system state variables are as following,

$$
X=\left[x, y, z, V_{x}, V_{y}, V_{z}\right]^{T}
$$

The moving speed of the mobile device is considered as a first-order Gauss Markov process.

$$
\left[\begin{array}{l}
\dot{x}(t) \\
\ddot{x}(t)
\end{array}\right]=\left[\begin{array}{ll}
0 & 1 \\
0 & -a_{x}
\end{array}\right]\left[\begin{array}{l}
x(t) \\
\dot{x}(t)
\end{array}\right]+\left[\begin{array}{l}
0 \\
1
\end{array}\right] \omega(t)
$$

In the formula, $\omega(t)$ is the white noise with the variance of $2 a_{x} \sigma_{x}^{2}$, the correlation coefficient $a_{x}$ is reciprocal with (t).

The system state equation is as following,

$$
\dot{X}(t)=A X(t)+W(t)
$$

where,

$$
A=\left[\begin{array}{cccccc}
0 & 0 & 0 & 1 & 0 & 0 \\
0 & 0 & 0 & 0 & 1 & 0 \\
0 & 0 & 0 & 0 & 0 & 1 \\
0 & 0 & 0 & -\frac{1}{\tau_{x}} & 0 & 0 \\
0 & 0 & 0 & 0 & -\frac{1}{\tau_{y}} & 0 \\
0 & 0 & 0 & 0 & 0 & -\frac{1}{\tau_{z}}
\end{array}\right]
$$

\subsection{Measurement Equation of Integrated Positioning System}

The single positioning system equation could be written as following

$$
y=H x+\varepsilon
$$

$y$ represents the $m \times 1$-dimensional measurement vector, $H$ represents the geometric observation matrix, ${ }^{\mathcal{E}}$ represents the measurement error vector, $x$ is the state vector of the user. Thus, the observation equations of BDS and GPS are respectively as following:

$$
\begin{aligned}
& y_{B D S}=H_{B D S} x_{B D S}+\varepsilon_{B D S} \\
& y_{G P S}=H_{G P S} x_{G P S}+\varepsilon_{G P S}
\end{aligned}
$$

Combining the formula of (11) and (12), we can conclude that the pseudo-range observation model of the integrated positioning system.

$\left\lfloor\begin{array}{l}y_{B D S} \\ y_{G P S}\end{array}\right\rfloor=\left\lfloor\begin{array}{lll}H_{B D S} & 1_{B D S} & 0_{B D S} \\ H_{G P S} & 0_{G P S} & 1_{G P S}\end{array}\right\rfloor x+\left\lfloor\begin{array}{l}\varepsilon_{B D S} \\ \varepsilon_{G P S}\end{array}\right\rfloor$

The observation equation of BDS/GPS integrated positioning system is obtained as following:

$$
Y_{\text {comb }}=H_{\text {comb }} \times X_{\text {comb }}+\varepsilon_{\text {comb }}
$$

The system observation equation is nonlinear.

$$
\begin{gathered}
Z=H X(t)+V(t) \\
V=\left[\begin{array}{ll}
\varepsilon_{1} & \varepsilon_{2}
\end{array}\right]^{T}
\end{gathered}
$$

In the equation, $\varepsilon_{1}$ and $\varepsilon_{2}$ represent the white Gaussian noise.

\section{TEST ${ }^{\prime}$ RESULTS AND ANALYSIS}

\subsection{Test Environment}

The positioning module is used in the test with a sports car in the outskirts of Beijing. The test road map is showed in Figure 1 . The test distance is $1349 \mathrm{~m}$, and the time is $568 \mathrm{~s}$ as well as the output of positioning result is $1 \mathrm{~Hz}$. 


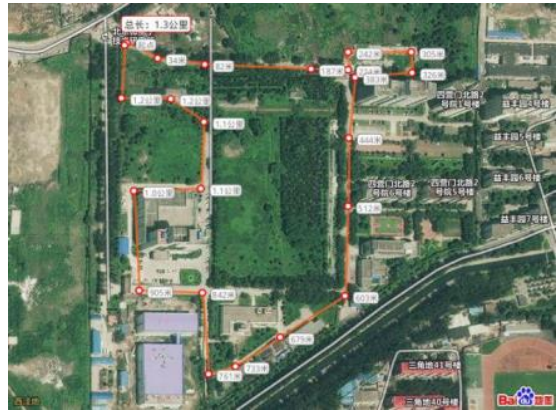

Figure1. Test road map

The paper uses BDS/GPS dual-mode compatible thumb receiver (MXTOS2-200) as the development module. The development module is shown in Figure2. The basic technical indicators of the receiver are shown in Table.

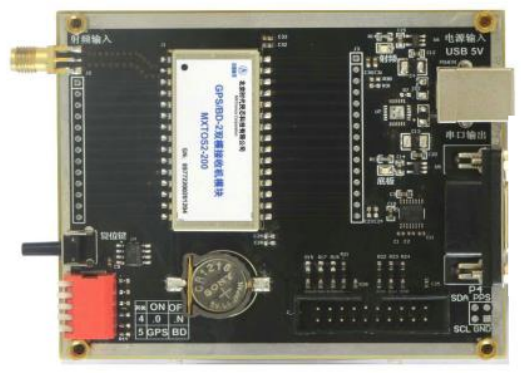

Figure 2: BDS/GPS dual-mode compatible thumb receiver

\begin{tabular}{|l|l|}
\hline \multicolumn{2}{|c|}{ Basic technical indicators } \\
\hline Received signal & $\begin{array}{l}\text { GPS L1 1575.42 MHz ; BD-2 B1 } \\
1561.098 \mathrm{MHz}\end{array}$ \\
\hline Channel number & $\begin{array}{l}\text { Programmable parallel channels of } \\
32 \text { independently }\end{array}$ \\
\hline $\begin{array}{l}\text { Output protocol } \\
\text { fomat }\end{array}$ & $\begin{array}{l}\text { NMEA0183, BUPro (special dual } \\
\text { mode communication protocol), } \\
\text { RINEX }\end{array}$ \\
\hline 1PPS output & Precision of 100 ns \\
\hline Data update rate & $1 \mathrm{~Hz}$ and 10 Hz (optional feature) \\
\hline
\end{tabular}

Table1: Basic technical indicators of the development module

\subsection{Result Analysis}

Figure 3 shows the detection probability's change with the satellite carrier to noise ratio and non-coherent integration times. The false alarm probability Pfa is 0.001 , the coherent integration time is $10 \mathrm{~ms}$, and the non-coherent integration frequency $\mathrm{N}$ is indefinite. When the signal carrier to noise ratio is fixed, if the non-coherent integration times are many enough, the detection probability is higher. The detection probability could be optimized with the increase of the non-coherent integration times.

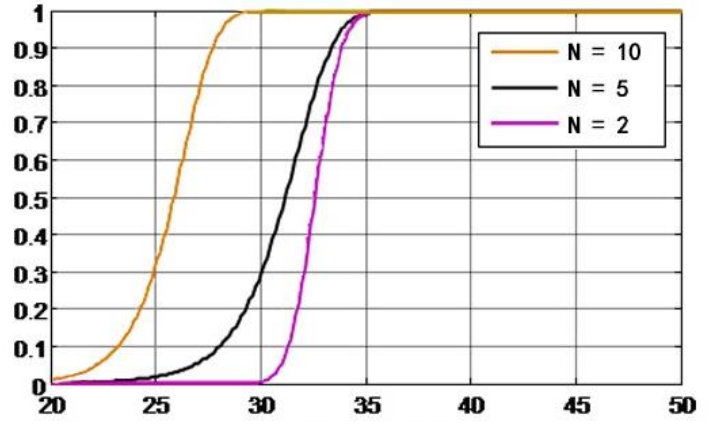

Figure 3: Change of detection probability with carrier to noise ratio

( $P_{f a}=0.001$, the coherent integration time is $10 \mathrm{~ms}$ )

As Figure 4 shows that we could capture satellite signal of $-130 \mathrm{dbm}$ through $10 \mathrm{~ms}$ coherent integration using the capture algorithms in this paper. In view of the weak signal environment, we are able to capture weak signal of $-140 \mathrm{dbm}$ through 10 times of non-coherent integration based on the coherent integration.
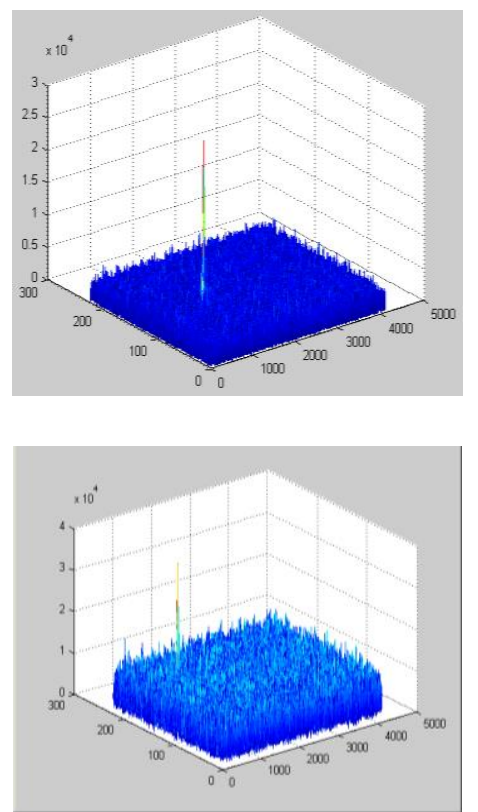

Figure4 : Signal acquisition correlation diagram

EKF, UKF and PF are used to deals with the test data respectively, and the following results are obtained. Figure5 represents the $\mathrm{X}$ direction positioning error comparison under three kinds of filter. Figure 6 represents the $\mathrm{Y}$ direction positioning error comparison and Figure 7 represents the $\mathrm{Z}$ direction positioning error comparison. 


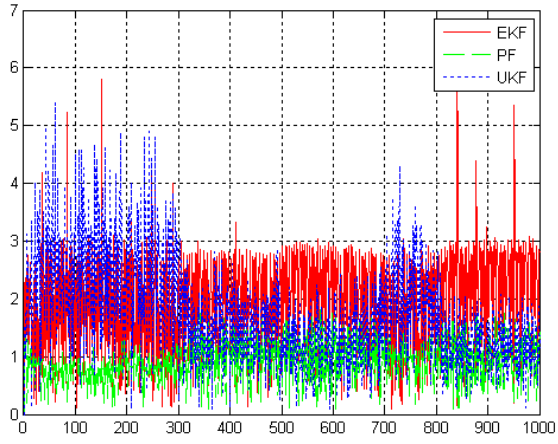

Figure5. X direction positioning error comparison under three kinds of filter

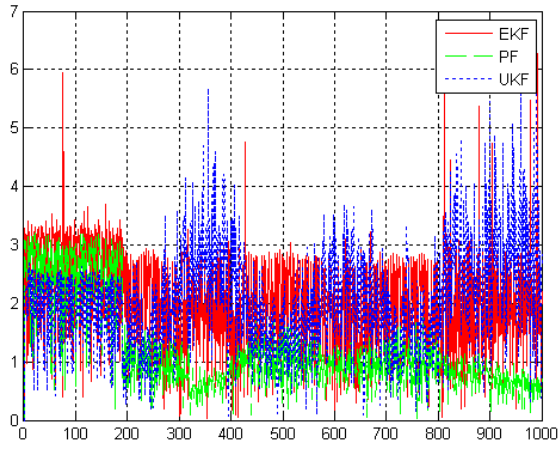

Figure6. Y direction positioning error comparison under three kinds of filter

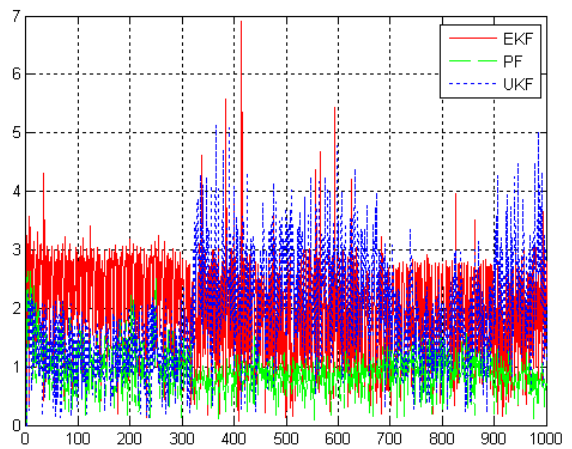

Figure7. Z direction positioning error comparison under three kinds of filter

The result analysis is based on 1000 epochs of positioning results. Figure 5, Figure 6 and Figure 7 are show that the positioning accuracy of PF is the best and the UKF is better than EKF.

For the $\mathrm{X}$ direction error statistics under three kinds of filter, Figure 8, Figure 9 and Figure 10 are given. The two different amplification level in the left show the horizontal positioning error, and the right show the accuracy of histograms and cumulative distribution as well as other related statistical characteristics.

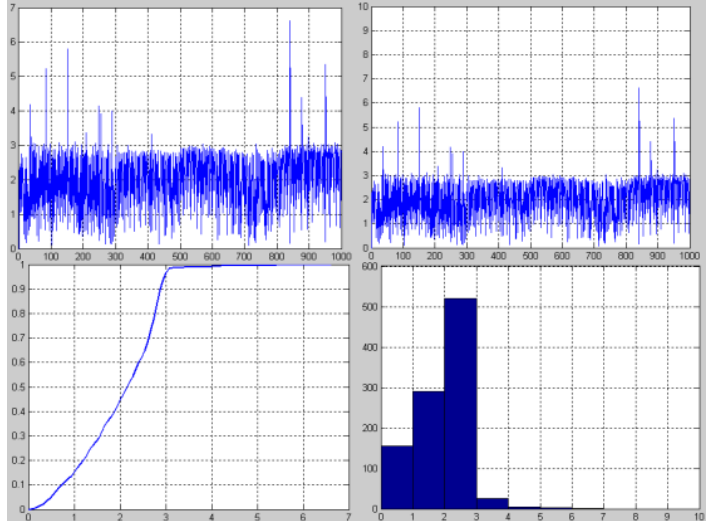

Figure8. horizontal direction of error statistics about the integrated positioning under $\mathrm{EKF}$

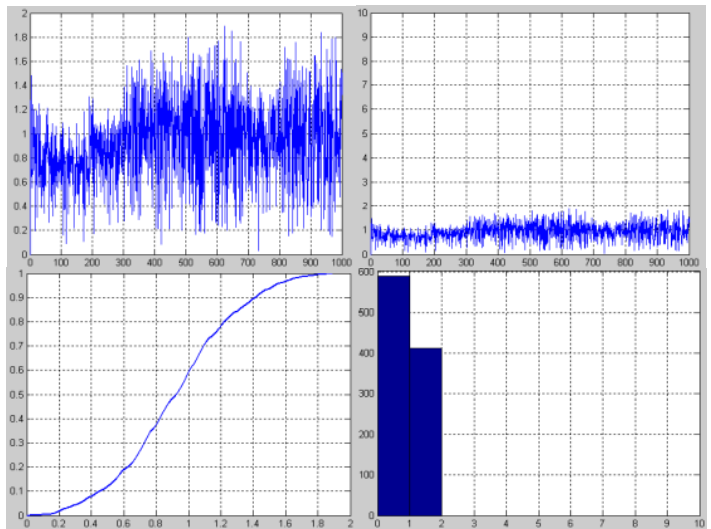

Figure9. horizontal direction of error statistics about the integrated positioning under UKF

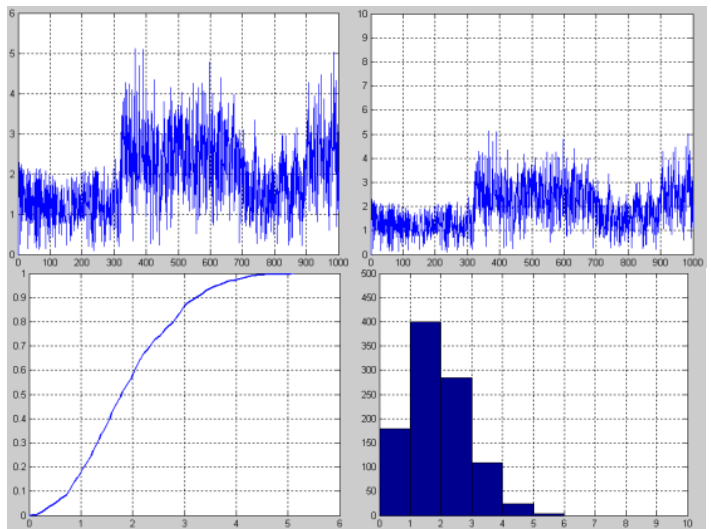

Figure 10. horizontal direction of error statistics about the integrated positioning under $\mathrm{PF}$

The error of the integrated positioning system about the horizontal direction under UKF and EKF and PF have been analyzed. Table 1 is the error analysis's statistical table. It shows that the result of PF is better than the other two. The reason may be that EKF and UKF are improved based on the linear Kalman filter algorithm for the nonlinear system. Therefore the filter is constrained by the linear Kalman filter algorithm, the system state should satisfy the Gaussian distribution. For the state model with non-Gaussian distribution, the filtering performance is not better enough. Instead, PF algorithm does not require the probability density of state variables with too many constraints. 


\begin{tabular}{|c|c|c|c|}
\hline & UKF & EKF & PF \\
\hline Usability & $\begin{array}{c}100 \% \\
(1000 / 1000)\end{array}$ & $\begin{array}{c}100 \% \\
(1000 / 1000)\end{array}$ & $\begin{array}{c}100 \% \\
(1000 / 1000)\end{array}$ \\
\hline $\begin{array}{c}\text { Maximu } \\
\text { s Error }\end{array}$ & $5.39 \mathrm{~m}$ & $6.6260 \mathrm{~m}$ & $1.8928 \mathrm{~m}$ \\
\hline $\begin{array}{c}\text { Average } \\
\text { Error }\end{array}$ & $1.75 \mathrm{~m}$ & $2.0034 \mathrm{~m}$ & $0.9205 \mathrm{~m}$ \\
\hline $\begin{array}{c}\text { Standard } \\
\text { Error }\end{array}$ & $0.92 \mathrm{~m}$ & $0.8492 \mathrm{~m}$ & $0.3573 \mathrm{~m}$ \\
\hline
\end{tabular}

Table 2. Error Analysis Statistical Table of Filter

\section{CONCLUSITION}

In this paper, the research of BDS/GPS integrated positioning method is carried out. The feasibility and stability of the method have also been verified by EKF, UKF and PF. The results of the test show that the positioning result accuracy of $\mathrm{PF}$ is the best, and the positioning result accuracy of UKF is better than EKF. As the algorithm used pseudo-range positioning model without carrier value observation, the accuracy of positioning result could be improved in high precision demand area with carrier value observation and the pseudo-range smoothing.

\section{ACKNOWLEDGEMENTS (OPTIONAL)}

The work presented in this paper is supported by NSFC (Natio nal Natural Science Foundation of China) (ID: U1533102 \& U 1433102).

\section{REFERENCES}

Park B, Kee C., 2001. Optimal Hatch Filter with a Flexible Smoothing Window Width, 592-602.

Sun Yanpeng, Zhang Yingshuo, Wang Ershen, Wang Bo, . 2011, BD-2/GPS Integrated Positioning System Design and Algorithm. In: Electronic Design Engineering, vol. 23, pp. 8083.

Zhang Baocheng, Yuan Yunbin, Jiang Zhen wei, 2015. Kalman Filter-based Single-baseline GNSS Data Processing without Pivot Satellite Changing. Acta Geodaetica et Cartographica Sinica, 44(9), pp. 958-964.

Liu Guixin, 2016. The Improved Adaptive Kalman Filter Algorithm. Electronic Design Engineering, 24(2), pp. 48-51.

Yin Jianjun, Zhang Jianqiu, Lin Qing, 2008. Unscented Kalman filter-Kalman filter Algorithm. System Engineering and Electronics., 30(4), pp. 617-620.

Zhou Qifan, Zhang Hai, Wang Yanran, 2015. A Redundant Measurement Adaptive Kalman Filter Algorithm, Acta Aeronautica et Astronautica Sinica, 36(5), pp. 1536-1605.

Dai Lu, Jin Guang, Chen Tao, 2008. Application of Adaptive Extended Kalman Filter in Spacecraft Attitude Determination
System, Journal of Jilin University(Engineering and Technology Edition), 38(2), pp. 466-470.

Zou Tao, Zhao Changsheng, Ding ZhenXiang, 2015. Unscented Kalman Filter of Nonlinear System with Colored Measurement Noise, Bulletin of Surveying and Mapping, pp.24-27.

Tang B, Dodds DE., 2006. Weak Signal GPS Synchronization for Locating In-building Cellular Telephones. Electrical and Computer Engineering, 13(4), pp. 928-931.

Stirling-gallacher R, Hulbert A,Povey GJ., 1996. A Fast Acquisition Technique for a Direct Sequence Spread Spectrum Signal in the Presence of a Large Doppler Shift[C]//Spread Spectrum Techniques and Applications Proceedings, 1996., IEEE 4th International Symposium On. Vol. 1. IEEE, pp. 156-160.

Grant P,Spangenberg S,Scott I,Mclaughlin S,Povey G,Cruickshank D., 1998. Doppler Estimation for Fast Acquisition in Spread Spectrum Communication Systems[C]//Spread Spectrum Techniques and Applications, 1998. Proceedings., 1998 IEEE 5th International Symposium on IEEE., pp.106-110.

O'driscoll C., 2007. Performance Analysis of the Parallel Acquisition of Weak GPS Signals. Department of Electrical and Electronic Engineering, UCC.

Tsui J., 2005. Fundamentals of Global Positioning System Receivers: a Software Approach. John Wiley \& Sons Inc.

Akos DM, Normark P, Lee J, Gromov KG, Tsui JB, Schamus J., 2000. Low Power Global Navigation Satellite System (GNSS) Signal Detection and Processing. Proceedings of the 13th International Technical Meeting of the Satellite Division of the Institute of Navigation (ION GPS 2000). pp 784-791. 